\title{
Early Changes of Brain Perfusion After Subarachnoid Hemorrhage - the Effect of Sodium Nitroprusside
}

\author{
M. KOLAR ${ }^{1}$, K. NOHEJLOVA ${ }^{2}$, J. MARES ${ }^{2}$, J. PACHL ${ }^{1}$ \\ ${ }^{1}$ Department of Anesthesiology and Critical Care Medicine, Third Faculty of Medicine, Charles \\ University, Prague, Czech Republic, ${ }^{2}$ Department of Normal, Pathological and Clinical Physiology, \\ Third Faculty of Medicine, Charles University, Prague, Czech Republic
}

Received March 27, 2016

Accepted October 26, 2016

\begin{abstract}
Summary
Causes of early hypoperfusion after subarachnoid hemorrhage

(SAH) include intracranial hypertension as well as vasoconstriction. The aim of the study was to assess the effect of intracerebroventricular (ICV) administration of sodium nitroprusside (SNP) on early hypoperfusion after SAH. Male Wistar rats (220-240 g) were used, SAH group received $250 \mu \mathrm{l}$ of fresh autologous arterial blood into the prechiasmatic cistern; sham-operated animals received $250 \mu$ of isotonic solution. Therapeutic intervention: ICV administration of $10 \mu \mathrm{g}$ SNP; $5 \mu \mathrm{l}$ $5 \%$ glucose (SNP vehicle) and untreated control. Brain perfusion and invasive blood pressure were monitored for $30 \mathrm{~min}$ during and after induction of SAH. Despite SNP caused increase of perfusion in sham-operated animals, no response was observed in half of SAH animals. The other half developed hypotension accompanied by brain hypoperfusion. There was no difference between brain perfusion in SNP-treated, glucose-treated and untreated $\mathrm{SAH}$ animals during the monitored period. We did not observe expected beneficial effect of ICV administration of SNP after SAH. Moreover, half of the SNP-treated animals developed serious hypotension which led to brain hypoperfusion. This is the important finding showing that this is not the option for early management in patient after SAH.
\end{abstract}

\section{Key words}

Subarachnoid hemorrhage • Sodium nitroprusside • Nitric oxide • Early brain injury • Rat

\section{Corresponding author}

K. Nohejlova, Department of Normal, Pathological and Clinical Physiology, Third Faculty of Medicine, Charles University, Prague,
Ke Karlovu 4, 12000 Prague, Czech Republic. Fax: (420) 224 923 827. E-mail: k.deykun@gmail.com

\section{Introduction}

Non-traumatic aneurysmal subarachnoid hemorrhage (SAH) causes both early and delayed changes of brain perfusion. The early brain injury (EBI) develops within first $72 \mathrm{~h}$ after aneurysm rupture (Cahill et al. 2006) and it is a major cause of mortality in such cases (Broderick et al. 1994). The arterial wall rupture causes extravasation of arterial blood into subarachnoid space under high pressure, leading to rapidly increased intracranial pressure (ICP) and reduced cerebral perfusion pressure (CPP) and cerebral blood flow (CBF) (Cahill et al. 2006, Sehba et al. 2012). Even animal models show that the early hypoperfusion is related not only to intracranial hypertension, but also to vasoconstriction associated with arterial blood present in subarachnoid space; reduction of $\mathrm{CBF}$ independent from increased ICP or decreased CPP was observed (Bederson et al. 1998, Schubert et al. 2009).

One of the mechanisms of early vasoconstriction is impairment of nitric oxide (NO)-mediated vasodilation. Decreased levels of NO metabolites were observed as early as $10 \mathrm{~min}$ after SAH induction (Sehba et al. 2000). The NO deficiency is probably caused by scavenging of NO (Sehba et al. 2012, Schwartz et al. 2000a,b). In physiologic conditions, the activity of NO is terminated by reaction with oxyhemoglobin which forms methemoglobin and nitrate (Helms and Kim-Shapiro 2013). Oxyhemoglobin in subarachnoid space is 
considered to be one of the major NO scavengers after SAH and thus it contributes to the early vasoconstriction (Li et al. 2016, Sehba et al. 2012).

Due to this mechanism, it is reasonable to consider NO donors for treatment of early vasoconstriction. In previous studies the cerebral vessels did respond to external administration of NO (Sehba et al. 1999, Sehba et al. 2007).

One of the NO donors studied in conditions of delayed cerebral vasospasm is sodium nitroprusside (SNP). The desired effect of SNP in the treatment of vasospasm is reached by release of $\mathrm{NO}$ at the adventitial side of brain circulation from the SNP molecule. Such a replacement of $\mathrm{NO}$ in the vascular wall attenuates the vasospasm after intrathecal SNP administration (Thomas et al. 1999). Beneficial effect of SNP was observed in several experimental (Egemen et al. 1993, Vatter et al. 2007) and clinical studies (Pachl et al. 2005, Raabe et al. 2002).

As far as we are aware, there were no studies with administration of sodium nitroprusside conducted under conditions of early brain injury. We hypothesized that intracerebroventricular (ICV) administration of SNP could be used as an effective treatment of the early vasospasm immediately after SAH. Because SNP is a potent vasodilator, the ICV route could help to reduce systemic side effects seen after intravenous administration, preserve its vasodilating effect on brain circulation and increase availability of NO in brain tissue.

\section{Materials and Methods}

All procedures were performed in accordance with the Ethical Guidelines of the Third Faculty of Medicine, Charles University, Prague, Czech Republic. They were in accordance with the Guidelines of the Animal Protection Law of the Czech Republic, which correspond with European Guidelines on Laboratory Animal Care. Special care was taken to minimize animal suffering.

Adult male Wistar rats (AnLab, Czech Republic; 220-240 g) were used. The animals were housed in cages by four under a 12-h light/dark cycle, with food and water ad libitum.

Surgical preparation of animals and induction of non-traumatic $S A H$

All procedures were conducted in deep general anesthesia (ketamine $100 \mathrm{mg} / \mathrm{kg}$ and midazolam $1.2 \mathrm{mg} / \mathrm{kg}$, intraperitoneally) with additional local anesthesia of the soft tissues of the head (trimecaine $1 \%$ $0.3 \mathrm{ml})$.

Femoral artery was cannulated to obtain blood sample for SAH induction as well as to allow invasive monitoring of arterial blood pressure. Mean arterial blood pressure (MAP) was calculated as $2 / 3$ of diastolic pressure $+1 / 3$ of systolic pressure. Rectal temperature was monitored and maintained at $37^{\circ} \mathrm{C}$ during the whole procedure.

The skull of the animals was exposed from soft tissues. Left lateral ventricle was cannulated for ICV administration access (Brain Infusion Kit 2, Alzet, USA). The catheter was inserted $-2 \mathrm{~mm}$ and $2 \mathrm{~mm}$ left from bregma and $3 \mathrm{~mm}$ deep from the skull surface into the cerebral ventricle (Paxinos and Watson 2013).

The skull above right hemisphere was mechanically thinned to allow the measurement of brain perfusion. Prechiasmatic cistern injection model of subarachnoid hemorrhage was used (Prunell et al. 2002). Briefly, a small borehole was drilled $7 \mathrm{~mm}$ rostral from the bregma. A needle with $26 \mathrm{G}$ cannula was inserted into prechiasmatic cistern at the $30^{\circ}$ angle anteriorly. In the SAH groups, $250 \mu \mathrm{l}$ of fresh non-heparinized autologous arterial blood (aBLD) was injected during $15 \mathrm{~s}$ immediately after withdrawal from femoral artery; sham animals received $250 \mu \mathrm{l}$ of isotonic solution (ISOSOL) of body temperature.

\section{Experimental groups}

Sixty four animals were divided into six groups: 1) SAH-induced animals (SAH; $n=12) ; 2) \mathrm{SAH}+\mathrm{ICV}$ administration of sodium nitroprusside (SAH-SNP; $\mathrm{n}=18) ; 3) \mathrm{SAH}+\mathrm{ICV}$ glucose $5 \%(\mathrm{SAH}-\mathrm{glc} ; \mathrm{n}=10)$; 4) sham-operated animals (sham, $n=8$ ); 5) sham + ICV SNP (sham-SNP; $\mathrm{n}=8$ ); and 6 ) sham + ICV glucose $5 \%$ (sham-glc; $\mathrm{n}=8$ ).

The SAH-SNP animals received $10 \mu \mathrm{g}$ of sodium nitroprusside (Sigma-Aldrich) in $5 \mu \mathrm{l}$ of $5 \%$ glucose solution $3 \mathrm{~min}$ after SAH induction. If secondary decrease of perfusion occurred after previous increase (decrease $>15 \%$ of perfusion after reaching peak level), second dose of SNP solution was administered. The solution of SNP was meticulously protected from light during preparation as well as during the whole experiment.

The SAH-glc group received $5 \mu \mathrm{l}$ of $5 \%$ glucose solution according to the same time pattern, the SAH group remained untreated. The corresponding shamoperated groups received the treatment in the same pattern. 
Evaluation of changes in the perfusion of cerebral cortex after $S A H$

Changes in the perfusion of cerebral cortex were assessed using Laser speckle-contrast analysis (LASCA). For detection and evaluation of changes, PeriCam PSI HR with PimSoft software (Perimed, Sweden) was used. The region of interest (ROI) for perfusion measurement was placed above the right hemisphere and it was limited by sagittal suture and lateral ridge, caudally by lambdoid suture and exceeded coronal suture for $3 \mathrm{~mm}$ in rostral direction. The tissue perfusion was measured in perfusion units (PU) standardized by manufacturer. Because the PUs cannot be converted to $\mathrm{ml} / \mathrm{min} / 100 \mathrm{~g}$, changes in perfusion were described in percentage of PU deviation from baseline record. The baseline levels were obtained from measurement performed in each animal during $1 \mathrm{~min}$ before induction of SAH. Framing speed was set as $1 \mathrm{image} / \mathrm{s}$ and the mean perfusion in the designated ROI was used for evaluation.

\section{Statistical analysis}

GraphPad Prism 6 (GraphPad Software, Inc., USA) was used for statistical evaluation. Changes in perfusion between groups were compared using two-way ANOVA for repeated measures with Bonferroni's post-test. Following changes in the perfusion of animals with SAH were analyzed using t-test: maximum values of perfusion which followed an initial drop after application of blood into prechiasmatic cistern; minimum values of perfusion decreased secondary after reaching maximum; as well as changes in mean arterial blood pressure and perfusion in groups treated with SNP.

Level of statistical significance was set to $\mathrm{p}<0.05$. All data are presented as mean \pm SEM.

\section{Results}

\section{Changes of brain perfusion}

Intracisternal administration of both aBLD and

ISOSOL was associated with significant decrease of brain perfusion.

The perfusion in the SAH group decreased to $23.3 \pm 4.5 \%$ (mean $\pm \mathrm{SE}$ ) of baseline values during the injection of aBLD. Three of 12 studied animals developed profound hypoperfusion leading to death within $5 \mathrm{~min}$ after the injection. In the remaining 9 animals, the perfusion recovered slowly after initial drop. Compared to control, the SAH group showed prolonged return towards baseline values and significantly reduced perfusion within first 5 min after SAH induction (Fig. 1).

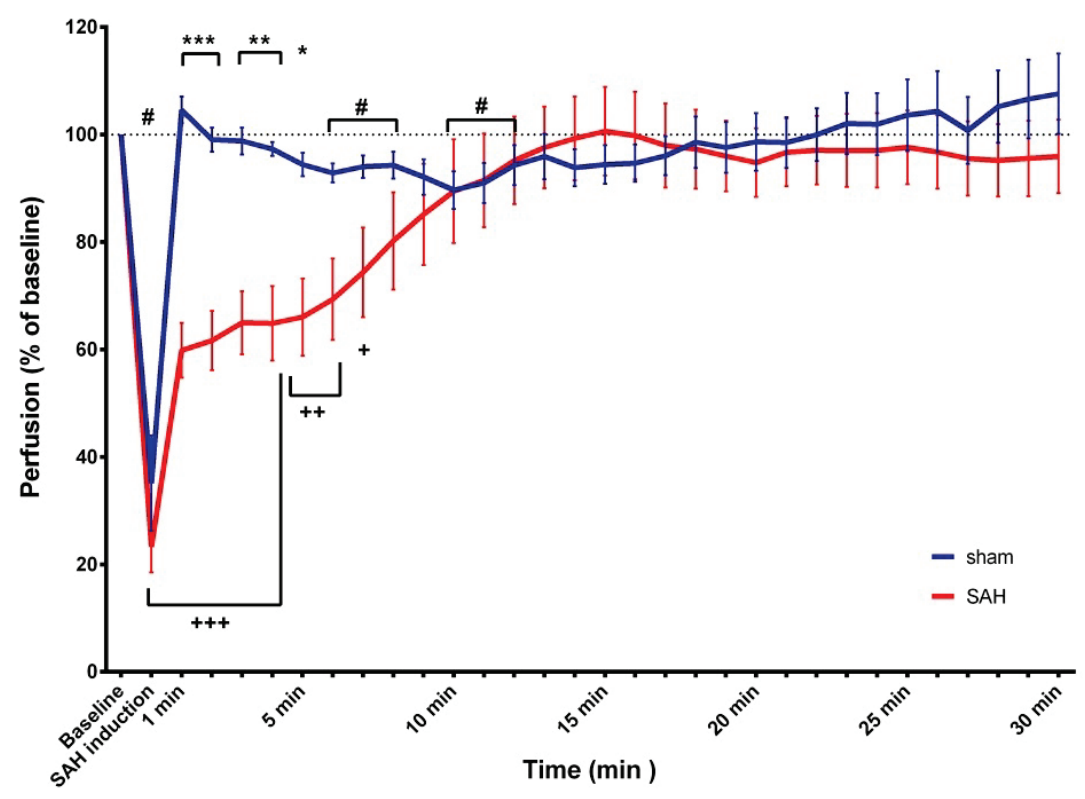

Fig. 1. Perfusion changes in SAH and shamoperated group. The return of perfusion towards baseline levels was prolonged in $\mathrm{SAH}$ group. The perfusion was significantly reduced during and first 7 min after $\mathrm{SAH}$ induction in SAH animals and at the time of intracisternal injection and 6-8 min and $10-12 \mathrm{~min}$ in sham-operated animals $(+, \# p<0.05, \quad++p<0.01, \quad+++p<0.001$ ， compared to baseline values). Compared to sham-operated animals, the perfusion was significantly lower first $5 \mathrm{~min}$ after $\mathrm{SAH}$ induction $(* \mathrm{p}<0.05, * * \mathrm{p}<0.01$, $* * * \mathrm{p}<0.001)$.

The perfusion changes in SAH animals showed biphasic time-course in 8 of 9 animals. After the injection, the perfusion recovered slowly reaching its peak in $14 \min 22 \mathrm{~s} \pm 2 \min 10 \mathrm{~s}$ and culminated at the level of $113 \pm 5.5 \%$ of baseline values. This was followed by another significant decrease $(\mathrm{p}<0.001$ and $\mathrm{p}<0.01$, compared to peak and baseline values, respectively) in $24 \min 52 \mathrm{~s} \pm 1 \mathrm{~min} 36 \mathrm{~s}$ at the level $86.4 \pm 3 \%$ of baseline after which the perfusion increased gradually. Similar pattern was observed in SAH-glc group, but only 
in half SAH-SNP animals.

In the sham group, the initial drop in perfusion was followed by short period of hyperperfusion after which the perfusion returned toward baseline level. The perfusion was significantly reduced during the time of intracisternal injection and 6-8 $\mathrm{min}$ and $10-12 \mathrm{~min}$ in sham-operated animals, compared to baseline values (Fig. 1).

The effect of intracerebroventricular administration of sodium nitroprusside

In the study group assigned for SNP treatment after SAH, one of 18 animals died before administration of SNP and 2 died after SNP administration. The response to SNP showed two different patterns. Eight animals showed no change of MAP and the time-course of brain perfusion did not differ significantly from SAH and SAH-glc groups. The other 7 animals developed serious systemic hypotension (drop in MAP up to $50 \%$ of pre-dosing values) which was accompanied with decrease of brain perfusion (Fig. 2). In this subgroup, the brain perfusion was significantly lower $1 \mathrm{~min}$ after the SNP administration, compared to non-hypotensive animals (Fig. 3).

Both normotensive and hypotensive animals showed increase of perfusion above the pre-dosing levels 3 min after the first SNP injection. Comparison with SAH-glc and SAH groups nevertheless shows that this increase of brain perfusion occurs in this time period independently from the SNP treatment.
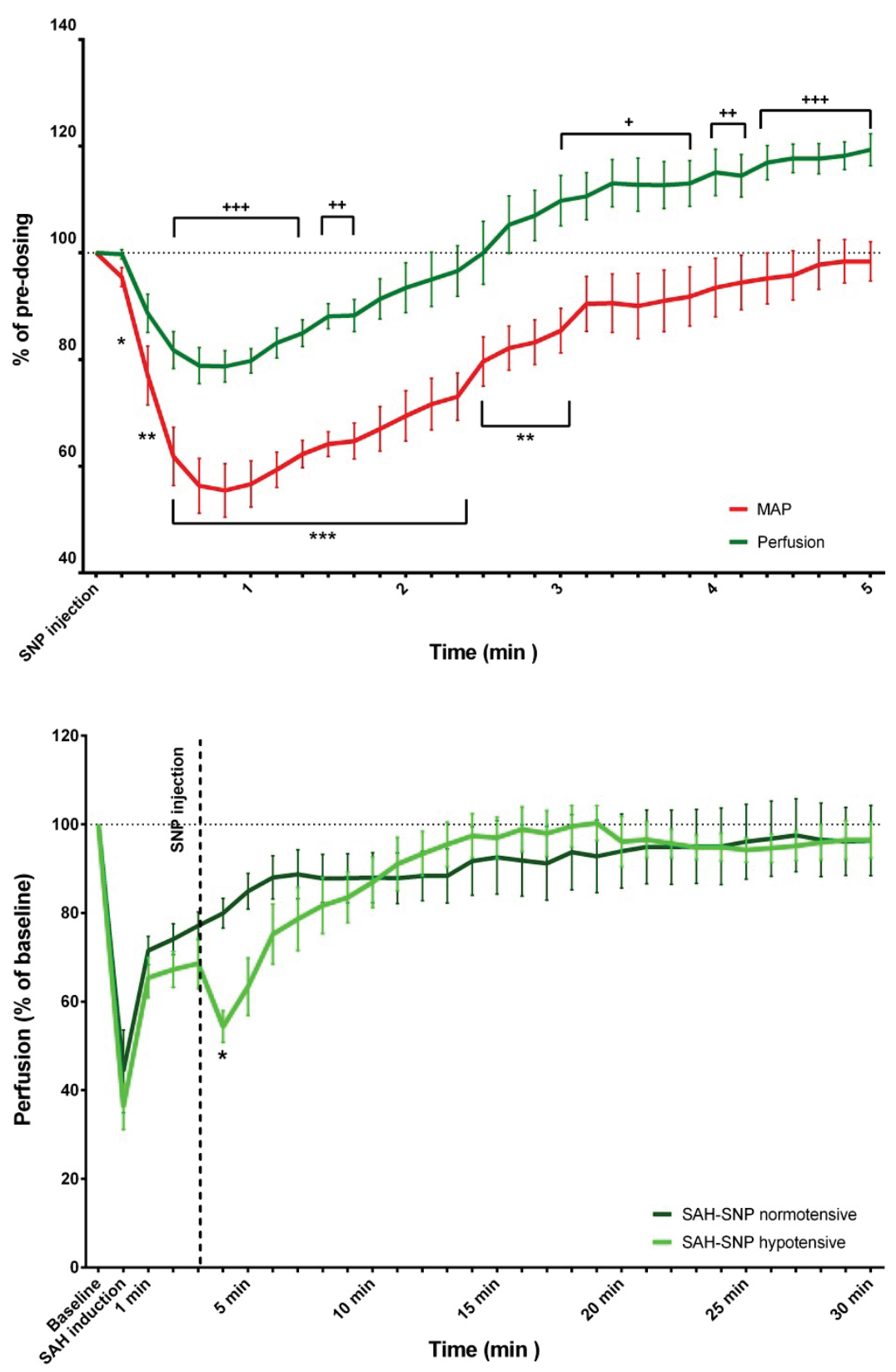

Fig. 2. Perfusion and MAP changes in SAH-SNP group after the first SNP dose. In 7 of 15 animals, SNP administration 3 min after SAH induction caused decrease of MAP, which corresponded with significant decrease of brain perfusion. Significant increase of brain perfusion above pre-dosing levels was observed $3 \mathrm{~min}$ after the injection; nevertheless this increase followed trends observed even in $\mathrm{SAH}$ and SAH-glc groups $\quad(*,+p<0.05, \quad * *,++p<0.01$, $* * *,+++p<0.001$, compared to pre-dosing levels). In contrast to other figures, baseline is set as pre-dosing level.

Fig. 3. Perfusion changes after SNP administration. Administration of SNP $3 \mathrm{~min}$ after SAH induction was followed by arterial hypotension and brain hypoperfusion in 7 of 15 animals. Brain perfusion was significantly lower $1 \mathrm{~min}$ after SNP injection in those animals which developed hypotension, compared to normotensive animals $(* p<0.05)$. 


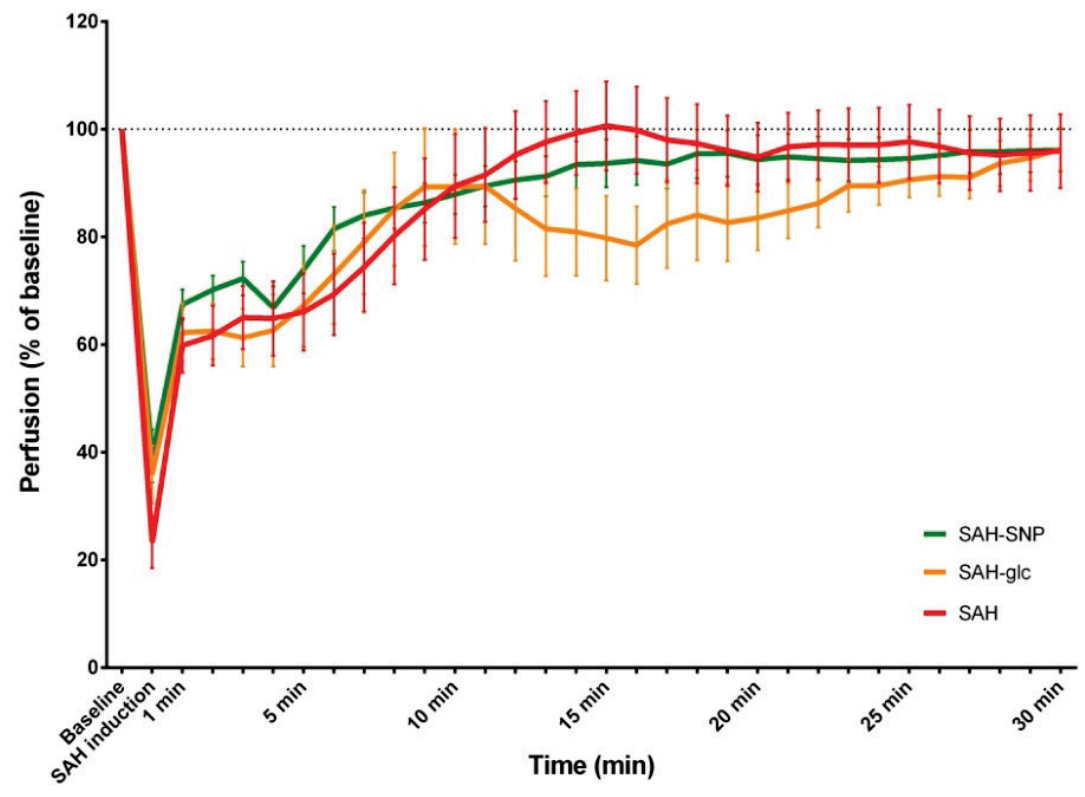

Fig. 4. Perfusion changes in groups with SAH. No statistical significance was observed between SAH, SAH-SNP and SAH-glucose groups.

Eight SNP-treated animals developed secondary decrease of perfusion after previous reaching of peak values. This decrease corresponded with biphasic timecourse of changes in perfusion seen in SAH group. These animals received another ICV dose of $10 \mu \mathrm{g}$ of SNP after secondary decrease between 11 and $25 \mathrm{~min}$. Sequentially a mild but significant decrease of MAP developed with insignificant decrease of perfusion. The perfusion increased $2 \mathrm{~min}$ after the second SNP injection and the raise above the baseline was significant $3 \mathrm{~min} 20 \mathrm{~s}$ after the injection (compared to pre-dosing values). This increase of perfusion however did not reach the values observed in sham-SNP animals.

Despite mild increase of the perfusion after the second dose of SNP, we did not observe any statistically significant increase of brain perfusion in SNP-treated animals, compared to SAH and SAH-glc group during the monitored period (Fig. 4). Also no difference was observed between animals which received 1 or 2 doses (data not shown).

The effect of intracerebroventricular administration of $5 \%$ glucose

One of $10 \mathrm{SAH}$-glc group animals died before and 2 died after the glucose injection. In 7 animals, the first dose of glucose did not cause any change of either mean arterial pressure or brain perfusion, compared to SAH group.

All 7 animals received second dose of glucose due to decrease of the perfusion after reaching the peak. The MAP remained unaffected after the injection. The injection of glucose caused transient increase of brain perfusion which was significant at $1.5 \mathrm{~min}$ (compared to pre-dosing values). In contrast to the SNP treated animals, the increase was observed within first $2 \mathrm{~min}$ after the injection. The perfusion in SAH-glc was significantly $(p<0.05)$ higher between $40 \mathrm{~s}$ and $1 \mathrm{~min}$, compared to SAH-SNP group.

\section{Sham-operated animals}

Animals in the sham-operated groups received ISOSOL instead of aBLD into the prechiasmatic cistern. Three minutes later, ICV injection of $5 \mu \mathrm{l}$ of either SNP or G5 \% solution was administered; one group received no treatment. Intracerebroventricular injection of SNP led to significant increase in brain perfusion above pre-dosing levels in 7 of 8 animals; this effect persisted till the end of the monitored period. In 1 animal, the increase of brain perfusion was achieved after repeated dose of SNP. The ICV injection of SNP was followed by mild decrease of MAP, nevertheless the brain perfusion increased in the same time. ICV injection of $5 \%$ glucose solution did not lead to any change of the perfusion (Fig. 5).

\section{Discussion}

Our study is the first to test the effect of intracerebroventricular administration of SNP in conditions of early hypoperfusion after subarachnoid hemorrhage.

Markedly reduced cortical perfusion is one of the initial consequences of SAH. The primary decrease of perfusion after intracisternal injection of both arterial 


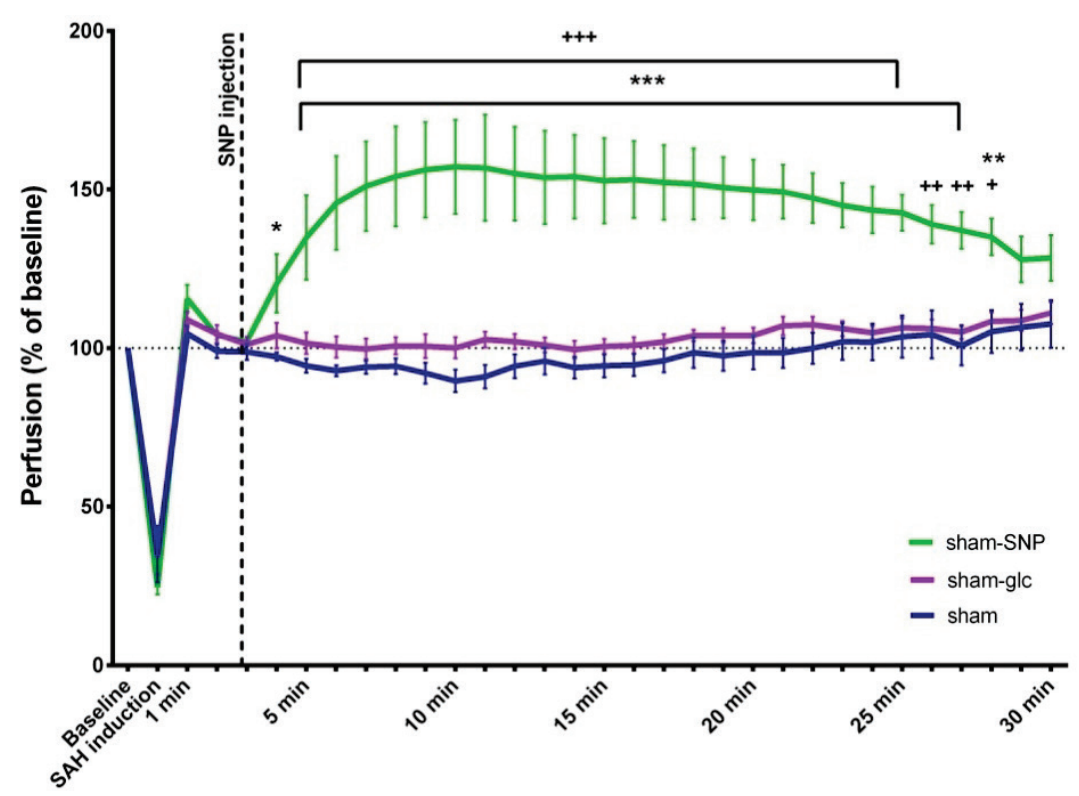

Time $(\min )$
Fig. 5. Perfusion changes in shamoperated groups. Intracerebroventricular administration of SNP to the sham-operated animals significantly increased the brain perfusion $(*,+p<0.05, \quad * *,++p<0.01$, $* * *,+++\mathrm{p}<0.001$; sham-SNP versus sham and sham-glc). Administration of $5 \%$ glucose solution caused no change of perfusion. blood and isotonic solution is caused by elevated ICP and decreased CPP (Bederson et al. 1995, Sehba et al. 2012). Nevertheless, animal studies show that the perfusion remains reduced even after CPP restore (Bederson et al. 1995). In clinical setting, measurement of CBF in patients suffering from aneurysmal $\mathrm{SAH}$ showed its reduction within the first $12 \mathrm{~h}$ after aneurysm rupture. That correlated with Hunt-Hess grade but not with ICP or CPP (Schubert et al. 2009). The protracted hypoperfusion after SAH has been described (Bederson et al. 1995, Schubert et al. 2008) and it can be attributed to early vasoconstriction occurring in presence of arterial blood in subarachnoid space.

It was suggested that hypoperfusion after $\mathrm{SAH}$ is caused both by intracranial hypertension and by disorders of brain macro- and microcirculation (Buhler et al. 2015, Sehba et al. 2007). Deficiency of NO in brain circulation due to its scavenging is considered to be a reason of early vasoconstriction (Sehba et al. 2012, Schwartz et al. 2000a,b). This is supported by finding of reduced levels of $\mathrm{NO}$ and its metabolites in the early phase after SAH (Sehba et al. 2000). In addition, brain vessels remain responsive to exogenous administration of NO (Sehba et al. 1999, Sehba et al. 2007). The supposed NO scavengers are hemoglobin (Sehba et al. 2000), superoxide anion (Kajita et al. 1994) or myeloperoxidase produced by neutrophils (Friedrich et al. 2011). Hemoglobin, a potent NO scavenger, is able to diffuse into the brain parenchyma, contact with microvessel pericytes and cause microvessel constriction (Li et al. 2016).
Nitric oxide donors treatment was studied in several experimental (Egemen et al. 1993, Vatter et al. 2007) and clinical (Pachl et al. 2005, Raabe et al. 2002) trials in conditions of delayed cerebral vasospasm.

Nitric oxide donors for prevention or treatment of EBI were used only in experimental settings: administration of an NO donor S-nitrosoglutathion (GSNO) was successfully used to increase CBF and preserved vascular wall integrity briefly after SAH induction (Sehba et al. 1999, Sehba et al. 2007). Another more common and affordable NO donor is sodium nitroprusside. The dose used in the present study was calculated according to the doses used in previous laboratory and clinical experiments and was adjusted on rats' body weight and volume of cerebrospinal fluid. The dose $10 \mu \mathrm{g}$ of SNP for bolus administration was proven as most effective in preliminary experiments.

In the present study, no favorable effect was observed after administration of SNP 3 min after SAH simulation. Half of the SNP-treated animals developed brain hypoperfusion instead. This can be related to either arterial hypotension or intracranial hypertension. Disturbance of autoregulation of brain perfusion is a frequent feature of intracranial pathologies. The brain perfusion depends on CPP and MAP in conditions of impaired autoregulation (Lang et al. 2003) and thus arterial hypotension after SNP injection can lower the brain perfusion substantially. Intracranial hypertension can also contribute to further reduction of perfusion. Different methods of SAH induction can produce 
different ICP profiles (Schwartz et al. 2000a,b); according to our previous yet unpublished findings, intracranial pressure $3 \mathrm{~min}$ after SAH induction is approx. 15-20 mm Hg in this model; similar results were obtained in another studies (Prunell et al. 2003). Injection of additional fluid volume (e.g. SNP) can increase ICP significantly in conditions of reduced brain compliance (Marmarou et al. 1975). Nevertheless this is doubted by fact that we observed no further brain perfusion decrease immediately after ICV injection of glucose solution.

Biphasic time course of the perfusion changes was observed in SAH animals. Reason for this pattern is uncertain. It can be related to the method of measurement of perfusion which includes both microcirculation and large pial vessels. Effect of SAH on vasoconstriction can differ between macro- and microcirculation (Sehba et al. 2012). The decrease of perfusion after previous peak can also reflect the changes in NO concentration in brain circulation (Sehba et al. 2000).

Mild increase of perfusion was observed after the second dose of SNP, which was administered after the second drop of perfusion. This increase did not reach the levels observed in sham-SNP group and there was no significant difference in perfusion between SAH-SNP group and $\mathrm{SAH}$ or $\mathrm{SAH}$-glc groups. In contrast, in sham-SNP group, the ICV administration of SNP led to prompt increase of brain perfusion which persisted till the end of the monitored period. This discrepancy in the effect of SNP between SAH and sham-operated group can have more reasons. Lack of effect of ICV administration of SNP can be caused by impaired distribution of the drug in subarachnoid space. The arteries of the circle of Willis are coated with the coagulum which can prevent from diffusion of the vasodilator and thus the concentration of NO in the vessel wall does not reach adequate level (Pluta et al. 2009).

Another possible explanation is that the dose leading to perfusion increase in sham-SNP animals can be insufficient in situation of $\mathrm{NO}$ depletion and scavenging which occurs after SAH. This can be supported by the finding of mild increase of brain perfusion after the second dose of SNP, which did not reach the levels of perfusion observed in sham-SNP animals. Variable response on SNP administration, mainly after the first dose of SNP, is consistent with marked interindividual variability which was observed also in clinical studies (Agrawal et al. 2009, Pachl et al. 2005).

So far, SNP was successfully used in experiments with delayed vasospasm - dilation of spastic vessels after SNP administration was observed in experiments on isolated arteries (Vatter et al. 2007) as well as in animal (Egemen et al. 1993) and clinical studies (Pachl et al. 2005, Raabe et al. 2002). The observed effect of intrathecal SNP administration in conditions of delayed cerebral vasospasm can be explained by the degradation of blood clot and improvement of SNP penetration into the target structures. The velocity of clot resolution was estimated as $10.8 \%$ of clot volume per day (Naff et al. 2001) and significant proportion of the hematoma is degraded at the time of occurrence of delayed cerebral vasospasm. Hemoglobin degradation products attribute to delayed vasospasm (Clark and Sharp 2006). Therefore, difference in mechanisms of vasoconstriction in the early and delayed phase after SAH can explain different finding in our model after SNP administration.

Hypotension after ICV injection of SNP was observed in significant proportion of study animals. This side effect was reported also in clinical studies (Agrawal et al. 2009). It can be attributed to direct effect on brain structures responsible for arterial blood pressure regulation - e.g. organum vasculosum laminae terminalis (Chang et al. 2001, Lin et al. 1999). Previous study demonstrated decrease of MAP after ICV injection of SNP (Lin et al. 1999), nevertheless the drop of MAP was less striking after dose equal to the dose used in our study.

The present study has some limitations, as well. Concentration of oxyhemoglobin in arterial blood can potentially affect the extent of the vasospasm, however neither hematocrit, nor $\mathrm{SpO}_{2}$ were measured in our study. The brain perfusion might also be influenced by anesthesia. Increase of $\mathrm{CBF}$ was described after ketamine; nevertheless the effect of ketamine on $\mathrm{CBF}$ was prevented by co-medication with midazolam (Strebel et al. 1995).

\section{Conclusions}

Intracerebroventricular administration of sodium nitroprusside did not produce expected effects in the conditions of early brain hypoperfusion in prechiasmatic cistern injection model of subarachnoid hemorrhage. It was also complicated with serious side effects - systemic hypotension that was connected with further decrease of cerebral perfusion pressure. The important outcome of the present study is that intracerebroventricular 
administration of sodium nitroprusside is not the option for early management in patient after subarachnoid hemorrhage.

\section{Conflict of Interest}

There is no conflict of interest.

\section{Acknowledgements}

This study was supported by the project \# NT144263/2013 from Internal Grant Agency of Ministry of Health of the Czech Republic, projects \# PRVOUK P34 and 260277/SVV/2016 from Charles University, Prague.

\section{References}

AGRAWAL A, PATIR R, KATO Y, CHOPRA S, SANO H, KANNO T: Role of intraventricular sodium nitroprusside in vasospasm secondary to aneurysmal subarachnoid haemorrhage: a 5-year prospective study with review of the literature. Minim Invasive Neurosurg 52: 5-8, 2009.

BEDERSON JB, GERMANO IM, GUARINO L: Cortical blood flow and cerebral perfusion pressure in a new noncraniotomy model of subarachnoid hemorrhage in the rat. Stroke 26: 1086-1092, 1995.

BEDERSON JB, LEVY AL, DING WH, KAHN R, DIPERNA CA, JENKINS AL 3RD, VALLABHAJOSYULA P: Acute vasoconstriction after subarachnoid hemorrhage. Neurosurgery 42: 352-362, 1998.

BRODERICK JP, BROTT TG, DULDNER JE, TOMSICK T, LEACH A: Initial and recurrent bleeding are the major causes of death following subarachnoid hemorrhage. Stroke 25: 1342-1347, 1994.

BUHLER D, AZGHANDI S, SCHULLER K, PLESNILA N: Effect of decompressive craniectomy on outcome following subarachnoid hemorrhage in mice. Stroke 46: 819-826, 2015.

CAHILL J, CALVERT JW, ZHANG JH: Mechanisms of early brain injury after subarachnoid hemorrhage. $J$ Cereb Blood Flow Metab 26: 1341-1353, 2006.

CHANG CP, PAN SP, LIN MT: A nitric oxide-dopamine link pathway in organum vasculosum laminae terminalis of rat brain exerts control over blood pressure. Br J Pharmacol 132: 1524-1530, 2001.

CLARK JF, SHARP FR: Bilirubin oxidation products (BOXes) and their role in cerebral vasospasm after subarachnoid hemorrhage. J Cereb Blood Flow Metab 26: 1223-1233, 2006.

EGEMEN N, TURKER RK, SANLIDILEK U, ZORLUTUNA A, BILGIC S, BASKAYA M, UNLU A, CAGLAR S, SPETZLER RF, MCCORMICK JM: The effect of intrathecal sodium nitroprusside on severe chronic vasospasm. Neurol Res 15: 310-315, 1993.

FRIEDRICH V, FLORES R, MULLER A, BI W, PEERSCHKE EI, SEHBA FA: Reduction of neutrophil activity decreases early microvascular injury after subarachnoid haemorrhage. J Neuroinflammation 8: 103, 2011.

HELMS C, KIM-SHAPIRO DB: Hemoglobin-mediated nitric oxide signaling. Free Radic Biol Med 61: 464-472, 2013.

KAJITA Y, SUZUKI Y, OYAMA H, TANAZAWA T, TAKAYASU M, SHIBUYA M, SUGITA K: Combined effect of L-arginine and superoxide dismutase on the spastic basilar artery after subarachnoid hemorrhage in dogs. J Neurosurg 80: 476-483, 1994.

LANG EW, LAGOPOULOS J, GRIFFITH J, YIP K, YAM A, MUDALIAR Y, MEHDORN HM, DORSCH NW: Cerebral vasomotor reactivity testing in head injury: the link between pressure and flow. J Neurol Neurosurg Psychiatry 74: 1053-1059, 2003.

LI Q, CHEN Y, LI B, LUO C, ZUO S, LIU X, ZHANG JH, RUAN H, FENG H: Hemoglobin induced NO/cGMP suppression deteriorate microcirculation via pericyte phenotype transformation after subarachnoid hemorrhage in rats. Sci Rep 6: 22070, 2016.

LIN MT, PAN SP, LIN JH, YANG YL: Central control of blood pressure by nitrergic mechanisms in organum vasculosum laminae terminalis of rat brain. Br J Pharmacol 127: 1511-1517, 1999.

MARMAROU A, SHULMAN K, LAMORGESE J: Compartmental analysis of compliance and outflow resistance of the cerebrospinal fluid system. J Neurosurg 43: 523-534, 1975.

NAFF NJ, WILLIAMS MA, RIGAMONTI D, KEYL PM, HANLEY DF: Blood clot resolution in human cerebrospinal fluid: evidence of first-order kinetics. Neurosurgery 49: 614-621, 2001.

PACHL J, HANINEC P, TENCER T, MIZNER P, HOUST'AVA L, TOMAS R, WALDAUF P: The effect of subarachnoid sodium nitroprusside on the prevention of vasospasm in subarachnoid haemorrhage. Acta Neurochir Suppl 95: 141-145, 2005. 
PLUTA RM, BUTMAN JA, SCHATLO B, JOHNSON DL, OLDFIELD EH: Subarachnoid hemorrhage and the distribution of drugs delivered into the cerebrospinal fluid. Laboratory investigation. J Neurosurg 111: 1001-1007, 1-4, 2009.

PRUNELL GF, MATHIESEN T, SVENDGAARD NA: A new experimental model in rats for study of the pathophysiology of subarachnoid hemorrhage. Neuroreport 13: 2553-2556, 2002.

PRUNELL GF, MATHIESEN T, DIEMER NH, SVENDGAARD NA: Experimental subarachnoid hemorrhage: subarachnoid blood volume, mortality rate, neuronal death, cerebral blood flow, and perfusion pressure in three different rat models. Neurosurgery 52: 165-176, 2003.

RAABE A, ZIMMERMANN M, SETZER M, VATTER H, BERKEFELD J, SEIFERT V: Effect of intraventricular sodium nitroprusside on cerebral hemodynamics and oxygenation in poor-grade aneurysm patients with severe, medically refractory vasospasm. Neurosurgery 50: 1006-1014, 2002.

SCHUBERT GA, SCHILLING L, THOME C: Clazosentan, an endothelin receptor antagonist, prevents early hypoperfusion during the acute phase of massive experimental subarachnoid hemorrhage: a laser Doppler flowmetry study in rats. J Neurosurg 109: 1134-1140, 2008.

SCHUBERT GA, SEIZ M, HEGEWALD AA, MANVILLE J, THOME C: Acute hypoperfusion immediately after subarachnoid hemorrhage: a xenon contrast-enhanced CT study. J Neurotrauma 26: 2225-2231, 2009.

SCHWARTZ AY, MASAGO A, SEHBA FA, BEDERSON JB: Experimental models of subarachnoid hemorrhage in the rat: a refinement of the endovascular filament model. J Neurosci Methods 96: 161-167, 2000a.

SCHWARTZ AY, SEHBA FA, BEDERSON JB: Decreased nitric oxide availability contributes to acute cerebral ischemia after subarachnoid hemorrhage. Neurosurgery 47: 208-215, $2000 \mathrm{~b}$.

SEHBA FA, DING WH, CHERESHNEV I, BEDERSON JB: Effects of S-nitrosoglutathione on acute vasoconstriction and glutamate release after subarachnoid hemorrhage. Stroke 30: 1955-1961, 1999.

SEHBA FA, SCHWARTZ AY, CHERESHNEV I, BEDERSON JB: Acute decrease in cerebral nitric oxide levels after subarachnoid hemorrhage. J Cereb Blood Flow Metab 20: 604-611, 2000.

SEHBA FA, FRIEDRICH V JR, MAKONNEN G, BEDERSON JB: Acute cerebral vascular injury after subarachnoid hemorrhage and its prevention by administration of a nitric oxide donor. J Neurosurg 106: 321-329, 2007.

SEHBA FA, HOU J, PLUTA RM, ZHANG JH: The importance of early brain injury after subarachnoid hemorrhage. Prog Neurobiol 97: 14-37, 2012.

STREBEL S, KAUFMANN M, MAITRE L, SCHAEFER HG: Effects of ketamine on cerebral blood flow velocity in humans. Influence of pretreatment with midazolam or esmolol. Anaesthesia 50: 223-228, 1995.

THOMAS JE, ROSENWASSER RH, ARMONDA RA, HARROP J, MITCHELL W, GALARIA I: Safety of intrathecal sodium nitroprusside for the treatment and prevention of refractory cerebral vasospasm and ischemia in humans. Stroke 30: 1409-1416, 1999.

VATTER H, WEIDAUER S, DIAS S, PREIBISCH C, NGONE S, RAABE A, ZIMMERMANN M, SEIFERT V: Persistence of the nitric oxide-dependent vasodilator pathway of cerebral vessels after experimental subarachnoid hemorrhage. Neurosurgery 60: 179-188, 2007. 